\title{
Improving clinical named entity recognition in Chinese using the graphical and phonetic feature
}

\author{
Yifei Wang $^{1 *}$, Sophia Ananiadou ${ }^{1}$ and Jun'ichi Tsujii ${ }^{1,2}$ \\ From 2018 International Workshop on Biomedical and Health Informatics (BHI) \\ Madrid, Spain. 3-6 December 2018
}

\begin{abstract}
Background: Clinical Named Entity Recognition is to find the name of diseases, body parts and other related terms from the given text. Because Chinese language is quite different with English language, the machine cannot simply get the graphical and phonetic information form Chinese characters. The method for Chinese should be different from that for English. Chinese characters present abundant information with the graphical features, recent research on Chinese word embedding tries to use graphical information as subword. This paper uses both graphical and phonetic features to improve Chinese Clinical Named Entity Recognition based on the presence of phono-semantic characters.

Methods: This paper proposed three different embedding models and tested them on the annotated data. The data have been divided into two sections for exploring the effect of the proportion of phono-semantic characters.

Results: The model using primary radical and pinyin can improve Clinical Named Entity Recognition in Chinese and get the F-measure of 0.712 . More phono-semantic characters does not give a better result.

Conclusions: The paper proves that the use of the combination of graphical and phonetic features can improve the Clinical Named Entity Recognition in Chinese.
\end{abstract}

Keywords: Text mining, Neural networks, Named entity recognition

This paper is an extended version of the workshop paper presented in BHI 2018 [1], discussions and new experiments about how phono-semantic characters will affect the result of applying the new method are stated in this paper.

\section{Background}

Named Entity Recognition (NER), as the name suggests, is a task to find the named entities from some given text. Named entities usually refer to some specific objects, such as persons and places. For the NER task in some languages using Latin alphabet like English, there are many available features to use, such as capital letters. But for Chinese, performing NER becomes difficult because there are no

${ }^{*}$ Correspondence: yifei.wang@manchester.ac.uk

'National Centre of Text Mining, University of Manchester, Manchester, UK Full list of author information is available at the end of the article spaces between words and there are no capital letters to identify special words. Furthermore, we can get both semantic and phonetic information from English words, while Chinese characters in machines alone do not provide any information on them as they are just a sequence of Unicode. So Chinese character embedding containing both semantic and phonetic information should help in the NER task.

A radical is the basic graphical component to form the character. For example, 病, which means illness, has two

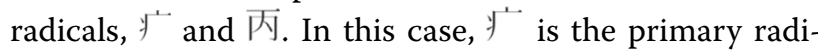
cal and suggests the meaning of the character is related to illness, and 丙 contains phonetic information suggesting the pronunciation of the character. The primary radical usually implies the meaning of a character. Table 1 shows some characters related to biomedicine with their meanings and primary radicals. It can be easily found that the

(c) The Author(s). 2019 Open Access This article is distributed under the terms of the Creative Commons Attribution 4.0 International License (http://creativecommons.org/licenses/by/4.0/), which permits unrestricted use, distribution, and reproduction in any medium, provided you give appropriate credit to the original author(s) and the source, provide a link to the Creative Commons license, and indicate if changes were made. The Creative Commons Public Domain Dedication waiver (http://creativecommons.org/publicdomain/zero/1.0/) applies to the data made available in this article, unless otherwise stated. 
Table 1 Phono-semantic characters in the biomedical domain

\begin{tabular}{|c|c|c|c|c|}
\hline Character & Pinyin & Primary Radical & Phonetic Radical & Pinyin of Phonetic Radica \\
\hline 病(illness) & bìng & 疤 (sickness) & $\overline{\text { 内 }}_{\text {(third) }}$ & bìng \\
\hline 庿(tuberculosis) & láo & 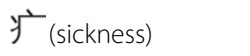 & 劳(labour) & láo \\
\hline 痛(pain) & tòng & 㡰 (sickness) & 角(path) & yǒng \\
\hline 肝(liver) & gān & 月(moon)/肉(meat) & $干_{(\mathrm{do})}$ & gàn \\
\hline 胸(chest) & xiōng & 月(moon)/肉(meat) & 匈(ancient form of 胸) & xiōng \\
\hline 脑(brain) & năo & 月(moon)/肉(meat) & $\overline{\mathbf{亠}}$ (bad luck) & xiōng \\
\hline
\end{tabular}

names of a disease share the same primary radical and the names of organs share same primary radical as well. In the case of organs, 月 is the simplification form of 肉.

Pinyin is a romanization system for Chinese, which can represent the pronunciation of a Chinese character in Latin letters. The pinyin of a character usually contains three parts: initial, final and tone. Initials and finals are similar to the consonant and vowel in English except there can be only one initial and one final in the pinyin of a character. There are five differny tones in the pinyin: flat tone ( $\left.{ }^{-}\right)$, rising tone ('), falling-rising tone $\left({ }^{\vee}\right)$, falling tone ( $\left.{ }^{\prime}\right)$ and neural tone ( ). As shown in Table 1 , the pinyin of 病 is bìng, where $b$ is the initial, ing is the final and ì shows that the tone is falling tone. 丙, the phonetic radical of 病 is also a Chinese character, whose pinyin is bing. In this example, only the tone is different. In some characters, such as 痛 in Table 1 , only the finals are the same in the original character and the phonetic radical, which are ong in this case. There is another case that the pinyin of the original character and the phonetic radical are completely different, but most characters sharing the same phonetic radical have similar pinyin. For example, 脑 shown in Table 1 has the same pinyin with 恼(nǎo) and 垴(nǎo), although their phonetic radical 㐫| is pronounced as xiōng.

But not all Chinese characters present the meaning with their primary radical and the pronunciation with the phonetic radical. Chinese characters that have primary radicals and phonetic radicals are called phonosemantic characters, more than $90 \%$ of Chinese characters are phono-semantic [2]. Some examples of non-phonosemantic characters in biomedical domain are shown in Table 2.

In Table 1, we can find that some of the biomedical characters are phono-semantic, containing primary radicals providing the semantic information and the phonetic radicals suggesting the pronunciation. So this paper attempts to explore whether primary radicals and the pinyin can help in Clinical NER in Chinese. While the characters in Table 2 do not have all the features, so applying the same method on these characters may not perform well, so another experiment is designed to explore how the proportion of phono-semantic characters will effect the result of using primary radicals and the pinyin.

Recently, with the development of deep learning, deep learning in Chinese NER has become popular. Wu et al. [3] applied the neural network with Conditional Random Field (CRF) to electronic health records and achieved the F-measure of 0.928. In the work of Peng et al. [4], word segmentation features were used to improve the Long Short-Term Memory-Conditional Random Field(LSTMCRF) model and got the F-measure of 0.484 when tested on social media data.

In English, a subword has the similar feature of the radical in Chinese because it contains some semantic information and suggests the meaning of the word. Some research has been made on subwords in English. In the research of Luong et al. [5], the words were split into several subwords, which are usually prefixes, suffixes and word roots, and the embedding of each subword will be composed to get the embedding of the word. The work of Bojanowski et al. [6] uses n-gram as the subword and trains the embedding for subwords.

In Chinese, radicals and other graphical features have been used in embedding training. In the work of $\mathrm{Yu}$ et al. [7], a new embedding method named JWE is introduced. In $J W E$, all radicals are regarded as subwords and following $C B O W$ [8] method. In the work of Cao et al. [9], $c w 2 v e c$ is introduced, where word2vec [8] is improved,

Table 2 Non-phono-semantic characters in the biomedical domain

\begin{tabular}{|c|c|c|c|c|}
\hline Character & Pinyin & Primary Radical & Other radical & Pinyin of Other Radica \\
\hline 胃(stomach) & wèi & 月(moon)/肉 (meat) & $\mathbb{H}_{(\text {(field) }}$ & tián \\
\hline 心(heart) & xīn & 心(heart) & - & - \\
\hline 害(harm) & hài & $\dot{\sim}$ (roof) & 丰(abundant) $\square_{\text {(mouth) }}$ & fēng kǒu \\
\hline
\end{tabular}


strokes were used to form $n$-grams as subwords. Another try in NER is from the work of Dong et al., which uses character embedding and radical embedding to get a better performance [10]. There are also some other attempts to catch the graphical features contained in Chinese characters. For example, Dai et al. trains the characters into glyph embeddings [11].

\section{Methods}

\section{Embedding models}

In the work of processing subwords in both Chinese and English $[6,7,9]$, the words are split into subwords first, and the subword embeddings are then trained to form the embedding of words. But when using primary radicals and pinyins, it is not a good idea to put them into one vocabulary to train because they are completely different things, thus it is meaningless to compare the similarity of primary radicals containing Chinese characters and pinyins containing Latin letters.

So the model proposed seeks to get the pretrained embeddings of primary radicals and pinyins separately and then combines them.

In the normal method of using the neural network in Chinese NER, the character embedding will only contain its character. Figure 1 shows the structure of Bi-LSTMCRF model [12]. In Fig. $1, C_{i}$ means the $i$ th character, $E_{i}$ means the embedding of the $i$ th character, and $T_{i}$ means the final tag of the character.

Different from simply looking for an embedding from the lookup layer, three different embedding models are proposed here to use the primary radical and other features in Fig. 2.

In the Radical+Character Model shown in Fig. 2a, the primary radical and the character itself are used to form the character embedding. The primary radical embedding $R E_{i}$ and character embedding $C E_{i}$ are obtained from the pretrained embeddings and form the final embedding $E_{i}$ for character $C_{i}$. This method is used to test how phonetic radicals affect the result.

In the Radical+Pinyin Model shown in Fig. 2b, the character embedding is formed by the primary radical and the pinyin. In Fig. $2 \mathrm{~b}, P_{i}, P E_{i}$ means the pinyin and pinyin embedding of character $C_{i}$, respectively. Because phonetic radicals usually do not provide tone information, only the initial and final are used for the pinyin. This method considers both semantic radicals and phonetic radicals.

In the Radical+Final Model shown in Fig. 2c, the character embedding comes from the primary radical and the final of pinyin. $F_{i}$ and $F E_{i}$ represents the final of pinyin and the embedding of the final of pinyin, respectively. This model is a modified version of Radical+Pinyin Model, as the initial information sometimes is not provided by the phonetic radical.

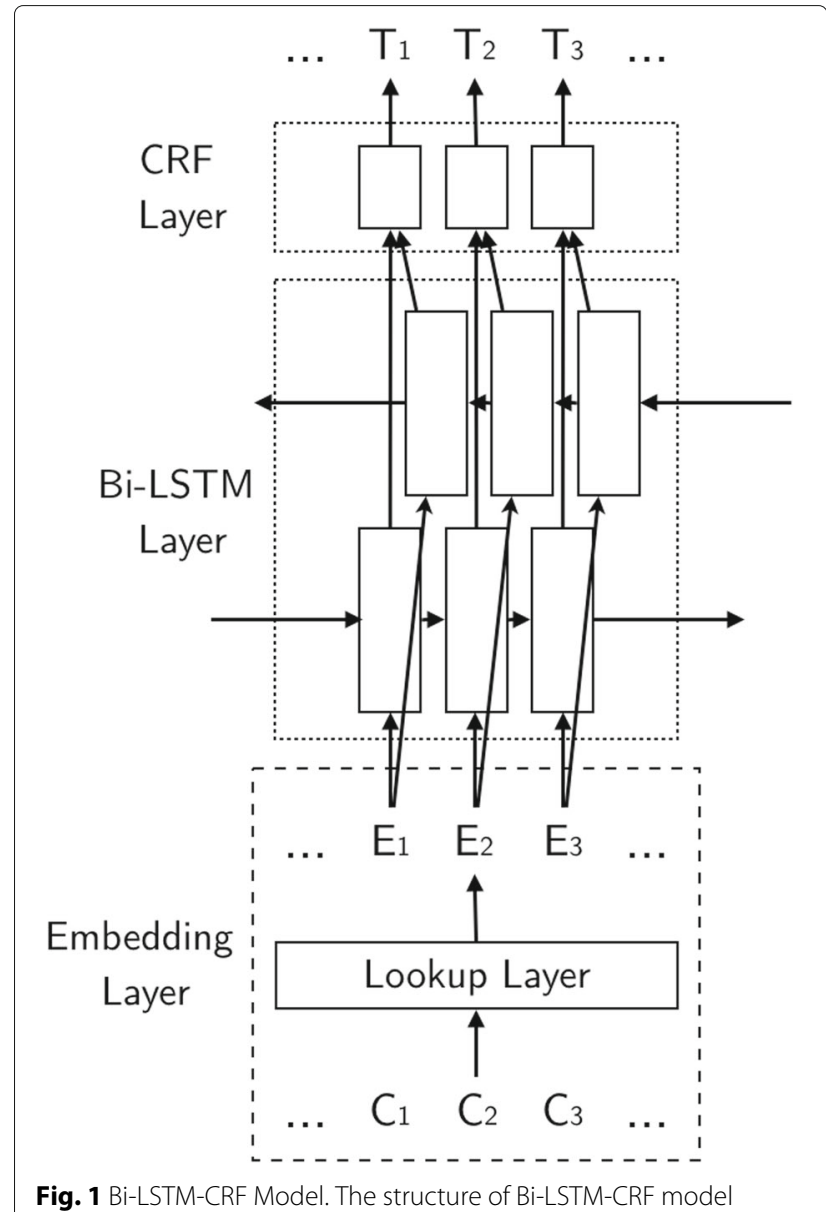

In all models, there is a composition layer to combine two different embeddings. A method to combine embeddings is proposed below:

$$
E_{i}=L W_{i} * L E_{i}+R W_{i} * R E_{i}+b_{i}
$$

In Equation $1, E_{i}$ is the final embedding, $L E_{i}$ and $R E_{i}$ represent the embedding to be composed, $L W_{i}$ and $R W_{i}$ are weight matrices and $b_{i}$ is the bias matrix. During the training, both weight matrices and bias matrices would be updated.

\section{Phono-semantic check}

Currently, there is not a common way to know whether a Chinese character is phono-semantic or not. People usually believe a character is phono-semantic when they can think of some other characters sharing the same phonetic radical and similar pronunciation. Shuowenjiezi, the dictionary published in the early 2nd century, states how each Chinese character is built, where phono-semantic is one of the building method. But it is not a proper resource for checking a phono-semantic character, as there are great 
a

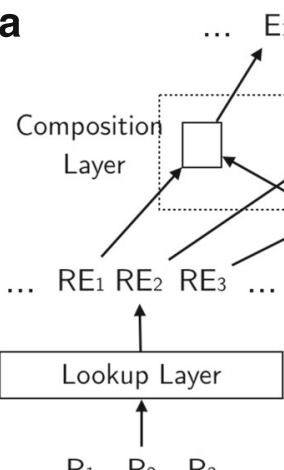

... $\mathrm{R}_{1}$

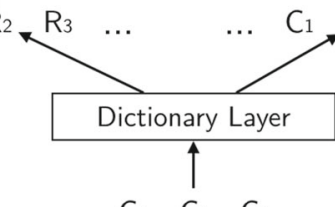

b $\begin{array}{llllllll}\ldots & C_{1} & C_{2} & C_{3} & \ldots\end{array}$

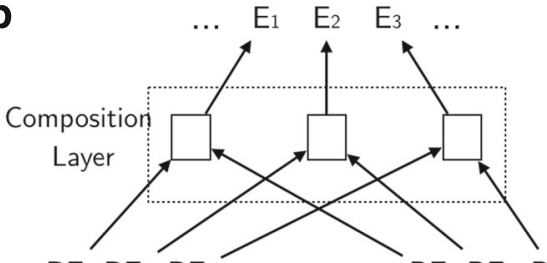
... $\mathrm{RE}_{1} \mathrm{RE}_{2} \mathrm{RE}_{3} \ldots \quad \ldots \mathrm{PE}_{1} \mathrm{PE}_{2} \mathrm{PE}_{3} \ldots$
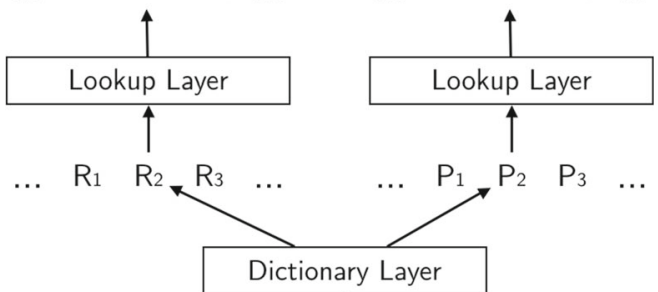

$\begin{array}{lllll}\ldots & C_{1} & C_{2} & C_{3} & \ldots\end{array}$

C

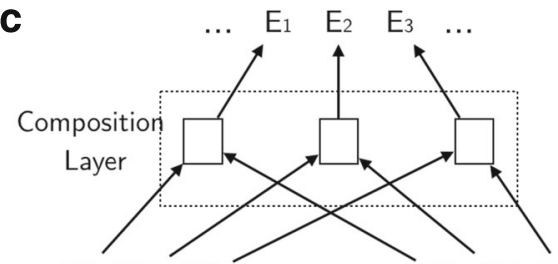

... $\mathrm{RE}_{1} \mathrm{RE}_{2} \mathrm{RE}_{3} \ldots \quad \ldots \mathrm{FE}_{1} \mathrm{FE}_{2} \mathrm{FE}_{3} \ldots$

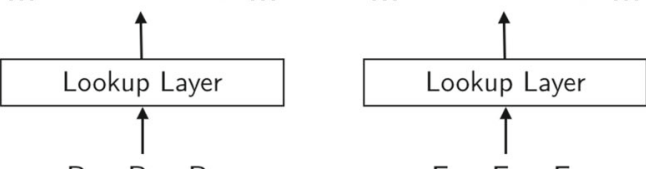

$\begin{array}{lllllllllll}\ldots & R_{1} & R_{2} & R_{3} & \ldots & \ldots & F_{1} & F_{2} & F_{3} & \ldots\end{array}$
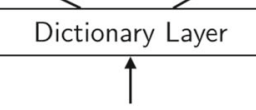

$\begin{array}{llllll}\ldots & C_{1} & C_{2} & C_{3} & \ldots\end{array}$

Fig. 2 Proposed Models. The structures of proposed embedding models. a Radical+Character Model. b Radical+Pinyin Model. c Radical+Final Model amount of characters built in later days by using phonosemantic method that are not included in this ancient dictionary.

Based on the feature of the phono-semantic character, one possible method to check whether a character is phono-semantic or not is to check the pinyin of the original character and all the pinyin of the forming radicals. If the original character shares the same final with one of the forming radicals, then the original character is a phono-semantic character.

However, there are some special cases. For example, the character 徒 (tú) has two radicals, 个 and 走, where 个 is the primary radical containing semantic information, and the pinyin of 走 is zóu. However, 徒 is a phono-semantic character, whose phonetic radical is t: (tǔ), one of the radicals of 走.

Ideographic Description Sequence (IDS) is a method to present how a Chinese-Japanaese-Korean (CJK) character is formed by using Ideographic Description Characters (IDC). For example, the IDS of 病 shown in Table 1 is 1 广丙, where the first character is an IDC, suggesting how the following two characters are used to form the original character. In this case, means that the first character should be on the top left of the second one. It is also possible to get the nested IDS of a Chinese character. In the case of 病, 丙 also has its IDS as - - 内, so that the nested IDS of 病 can be presented as 1. via viewing all nested IDS.

The pseudo code of the method used to check whether a character is phono-semantic or not is shown in Algorithm 1. IDS() is the function to get the IDS of a character, if the character cannot be divided anymore, the character itself will be returned. Final() is the function to get the final of the pinyin of the character.

\section{Data}

The data used in the experiment are provided in the China Conference on Knowledge Graph and Semantic Computing (CCKS) in 2017, which collects different clinical texts and contains 280,913 characters. The corpus uses $\mathrm{BIO}$ format to label five different named entity types: body part (BOD), symptom (SYM), disease (DIS), experiment (EXP) and treatment (TRE). A 5-cross-validation is performed to make the experiment.

Table 3 Sections of data for phono-semantic test

\begin{tabular}{lll}
\hline & Phono-semantic\% & Unique Phono-semantic\% \\
\hline A & 36.6 & 46.2 \\
B & 33.0 & 43.9 \\
\hline
\end{tabular}


Table 4 F-measure of different models on CCKS data

\begin{tabular}{lllllllll}
\hline Model & JWE & cw2vec & $R+C$ & $R+P$ & $R+F$ & $R+C($ Sum $)$ & $R+P($ Sum $)$ & $R+F($ Sum) \\
\hline BOD & 0.614 & 0.595 & 0.666 & 0.688 & 0.661 & 0.660 & 0.678 & 0.652 \\
SYM & 0.716 & 0.682 & 0.724 & 0.746 & 0.735 & 0.725 & 0.734 & 0.727 \\
DIS & 0.623 & 0.528 & 0.677 & 0.777 & 0.629 & 0.666 & 0.751 & 0.574 \\
EXP & 0.706 & 0.666 & 0.715 & 0.720 & 0.723 & 0.721 & 0.710 & 0.721 \\
TRE & 0.516 & 0.513 & 0.672 & 0.618 & 0.549 & 0.667 & 0.616 & 0.519 \\
ALL & 0.669 & 0.635 & 0.696 & $\mathbf{0 . 7 1 2}$ & 0.695 & 0.696 & 0.702 & 0.687 \\
\hline
\end{tabular}

The highest F-measure among all models is in bold

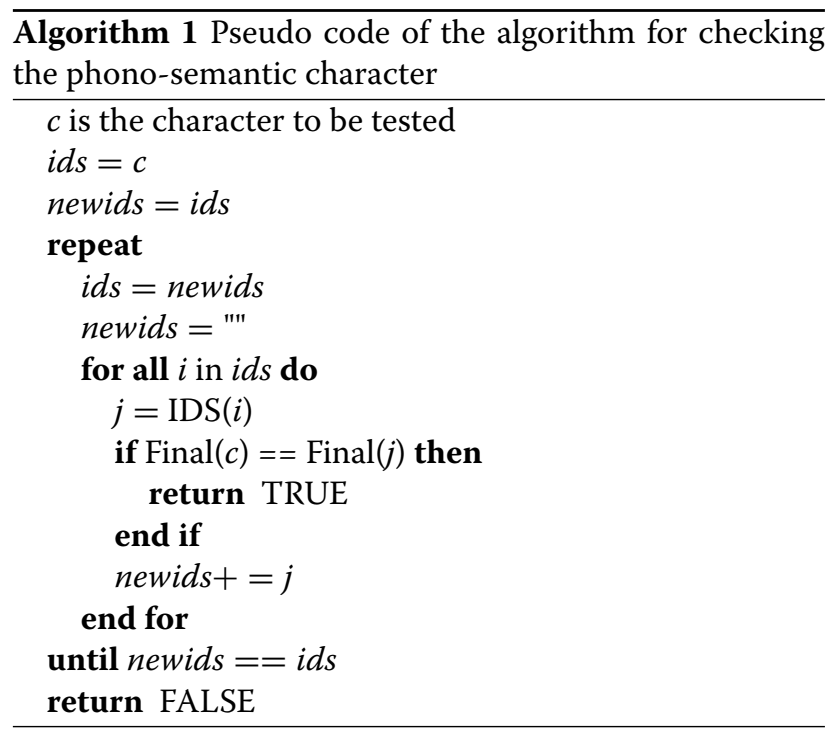

To explore how the proportion of the phono-semantic characters affects the results, the data have been split into two sections. The percentages of the phono-semantic characters in each data are calculated. The data will be put in section $\mathrm{A}$ if the percentage is larger than the median. Otherwise, the data will be put in section $B$.

The details of two sections are shown in Table 3. The Phono-semantic\% column shows the percentage of the phono-semantic characters in all characters, and The Unique Phono-semantic\% column shows the percentage of the unique phono-semantic characters in all unique characters.

The neural network model used for different embedding models is Bi-LSTM-CRF [12]. As only character embedding can be gained from the models proposed, the experiment here is done on character-based NER. The use of character-based NER will also prevent the problem of Chinese Word Segmentation.

To better catch the clinical texts, the pretrained embeddings are trained in a certain domain by using the Chinese Wikipedia under the category Medicine [13] and the category Biology [14] and their nested subcategories. Word2vec [15] package is used for pretraining character embedding, radical embedding and pinyin embedding.
To compare with other methods, the JWE model $[7,16]$ and the $c w 2 v e c$ model $[9,17]$ mentioned in Background part have also been tested. As both models are used for word embedding, each character in Wikipedia data is regarded as a word to train the character embedding. For a fair comparison, the same parameters including the learning rate, window size, embedding size have been used. The best model will be tested for the effect of the proportion of the phono-semantic characters.

The primary radical and pinyin information are gained from the Unihan database [18], IDS of all characters are gained from CHISE project [19], which annotates the IDS of most CJK characters.

\section{Results \\ Model comparison}

Table 4 shows the results of different models on CCKS data. $\mathrm{R}+\mathrm{C}, \mathrm{R}+\mathrm{P}$ and $\mathrm{R}+\mathrm{F}$ stand for the model in Fig. $2 \mathrm{a}, \mathrm{b}$ and c, respectively. The model with (Sum) is a simplified version, where $L W_{i}$ and $R W_{i}$ are fixed as 1 and $b_{i}=0$.

\section{Phono-semantic Proportion}

Based on the result of model comparison, $\mathrm{R}+\mathrm{P}$ model is used for exploring the affect of phono-semantic proportion. The result is shown in Table 5 .

\section{Discussion}

\section{Model comparison}

In Table 4, it can be clearly found that Model Radical+Pinyin gives the best performance, especially on disease type. It is proved that the use of the graphical and

Table 5 F-measure of different sections using model R+P

\begin{tabular}{lll}
\hline & Section A & Section B \\
\hline BOD & 0.620 & 0.651 \\
SYM & 0.691 & 0.749 \\
DIS & 0.584 & 0.680 \\
EXP & 0.671 & 0.753 \\
TRE & 0.578 & 0.475 \\
ALL & 0.650 & 0.712 \\
\hline
\end{tabular}


Table 6 Radical occurrence in named entities

\begin{tabular}{|c|c|c|c|c|c|c|c|c|c|}
\hline \multicolumn{2}{|l|}{ BOD } & \multicolumn{2}{|l|}{ SYM } & \multicolumn{2}{|l|}{ DIS } & \multicolumn{2}{|l|}{ EXP } & \multicolumn{2}{|l|}{ TRE } \\
\hline Radical & Occurrence & Radical & Occurrence & Radical & Occurrence & Radical & Occurrence & Radical & Occurrence \\
\hline 肉 & & 肉 & & 肉 & & 肉 & & 水 & \\
\hline (meat) & $17.2 \%$ & (meat) & $15.0 \%$ & (meat) & $11.0 \%$ & (meat) & $14.3 \%$ & (water) & $8.1 \%$ \\
\hline$\square$ & $6.5 \%$ & & $13.0 \%$ & & $8.4 \%$ & & $5.5 \%$ & & $5.1 \%$ \\
\hline (mouth) & & (mouth) & & (heart) & & (wood) & & (silk) & \\
\hline $\begin{array}{l}\text { 又 } \\
\text { (again) }\end{array}$ & $4.7 \%$ & $\begin{array}{l}\text { 㡰 } \\
\text { (sickness) }\end{array}$ & $10.4 \%$ & $\begin{array}{l}\text { 㡰 } \\
\text { (sickness) }\end{array}$ & $7.1 \%$ & $\begin{array}{l}\square \\
\text { (mouth) }\end{array}$ & $5.3 \%$ & $\begin{array}{l}\text { 肉 } \\
\text { (meat) }\end{array}$ & $4.8 \%$ \\
\hline $\begin{array}{l}\text { 人 } \\
\text { (human) }\end{array}$ & $4.6 \%$ & $\begin{array}{l}\text { 又 } \\
\text { (again) }\end{array}$ & $4.9 \%$ & $\begin{array}{l}\text { 系 } \\
\text { (silk) }\end{array}$ & $4.7 \%$ & $\begin{array}{l}\text { 人 } \\
\text { (human) }\end{array}$ & $5.1 \%$ & $\begin{array}{l}\text { 岖 } \\
\text { (grass) }\end{array}$ & $4.7 \%$ \\
\hline $\begin{array}{l}\text { 邑 } \\
\text { (state) }\end{array}$ & $4.1 \%$ & $\begin{array}{l}\text { 大 } \\
\text { (big) }\end{array}$ & $4.2 \%$ & $\begin{array}{l}\text { 火 } \\
\text { (fire) }\end{array}$ & $4.6 \%$ & $\begin{array}{l}\text { 心 } \\
\text { (heart) }\end{array}$ & $3.4 \%$ & $\begin{array}{l}\text { 手 } \\
\text { (hand) }\end{array}$ & $3.7 \%$ \\
\hline
\end{tabular}

phonetic feature of a character can be used for character embedding in an NER task as it has a better NER performance.

The reason that both JWE and cw2vec models do not have a good performance may be that they are designed for word embeddings. When applying them to character embedding, there might be some unnecessary operations and need more iterations for training.

The models that only sum two embeddings together have a slightly worse performance as $L W_{i}, R W_{i}$ and $b_{i}$ are fixed, which is evidence that the learning process in the composition layer is necessary.

Tables 6 and 7 show the radicals occurring frequently in CCKS dataset. In both SYM and DIS types, there are some primary radicals occurring a lot, it might be the reason the model performs well in these two named entity types.

\section{Phono-semantic proportion}

Based on the method how two sections are built and the phono-semantic percentage shown in Table 3, section A has more phono-semantic characters than section $\mathrm{B}$ does. If the model highly relies on phono-semantic characters, the model should have better result on section A. However, as shown in Table 5, the model performs better on section $\mathrm{B}$.

Two sections have been reviewed, and new statistical result is shown in Table 8 . The table is similar to Table 3,

Table 7 Overall radical occurrence in ccks data

\begin{tabular}{ll}
\hline Radical & Occurrence \\
肉(meat) & $6.1 \%$ \\
口(mouth) & $4.9 \%$ \\
木(wood) & $3.8 \%$ \\
人(human) & $3.3 \%$ \\
又(again) & $3.2 \%$
\end{tabular}

except that only named entity characters are considered. It shows that section $B$ has more unique phono-semantic characters in named entities, which might be why the model has better performance on section $B$.

In both Tables 3 and 8, the proportion of phonosemantic characters is much smaller than the $90 \%$ stated by Boltz [2]. It is because the method for checking phonosemantic characters is not perfect yet, for example, 脑 shown in Table 1 will not be considered as phonosemantic in this method. It might be another possible reason that such the result comes out.

It is also possible that the proportion of phonosemantic characters do not affect the performance of the embedding model. The reason that phonetic features can improve the result may be that a large amount of biomedical terms are translated with similar pronunciations, and models with phonetic features may be able to catch them. Some translated examples are shown in Table 9. More experiments are needed to find out the real reason.

\section{Conclusion}

This work proposes a method to use the graphical feature and phonetic feature in Clinical NER in Chinese. Based on the experiment on Bi-LSTM-CRF, the model using the primary radical feature and pinyin can improve the performance. The F-measure has been improved by 0.043 when using model $\mathrm{R}+\mathrm{P}$ compared to JWE.

But the work has the limitation that only characterbased NER is tested, and some work should be done for word-based NER as well. It is also necessary to develop

Table 8 Named entities in two sections

\begin{tabular}{lll}
\hline Named Entity Phono-semantic\% & $\begin{array}{l}\text { Named Entity Unique } \\
\text { Phono-semantic\% }\end{array}$ \\
\hline A 45.3 & 49.1 \\
B $\quad 41.9$ & 50.5 \\
\hline
\end{tabular}


Table 9 Translated biomedical terms

\begin{tabular}{lll}
\hline English & Chinese & Pinyin \\
\hline Parkinson's disease & 帕金森氏症 & pà jīn sēn shì zhèng \\
Aspirin & 阿司匹林 & à sī pì lín \\
\hline
\end{tabular}

a better composition layer. A complex composition layer may result in good performance but require much more time for training, which is also a problem.

The results of exploring the affect of the proportion of the phono-semantic characters suggest that the new embedding model has better performance on lower proportion data. Some possible reasons are stated, and new experiments should be carried out to verify them.

\section{Abbreviations \\ BOD: Body Part; CBOW: Continuous Bag-Of-Words; CJK:}

Chinese-Japanese-Korean; CRF: Conditional Random Field; DIS: Disease; EXP: Experiment; IDC: Ideographic Description Characters; IDS: Ideographic Description Sequence; JWE: Joint Learning Word Embedding; LSTM-CRF: Long Short-Term Memory-Conditional Random Field; NER: Named Entity Recognition; SYM: Symptom; TRE: Treatment

\section{Acknowledgements}

We thank all the anonymous reviewers for the helpful comments.

\section{About this supplement}

This article has been published as part of BMC Medical Informatics and Decision Making Volume 19 Supplement 7, 2019: Supplement special Issue on Biomedical and Health Informatics. The full contents of the supplement are available online at https://bmcmedinformdecismak.biomedcentral.com/articles/ supplements/volume-19-supplement-7.

\section{Funding}

The publication cost of this article was funded by School of Computer Science Kilburn Overseas Fees Bursary from the University of Manchester.

\section{Authors' contributions}

YW designs the models, performs the experiments and writes the paper. SA guides the project and helps to modify the paper. JT advises on the model. All authors read and reviewed the final manuscript. All authors read and approved the final manuscript

\section{Availability of data and materials}

The ccks dataset analysed during the current study is adopted from the Chinese EMR NER task in China Conference on Knowledge Graph and Semantic Computing in 2017(ccks2017), but restrictions apply to the availability of these data,which were used under license for the current study, and so are not publicly available.

\section{Ethics approval and consent to participate}

Not applicable

\section{Consent for publication}

Not applicable

\section{Competing interests}

The authors declare that they have no competing interests.

\section{Author details}

${ }^{1}$ National Centre of Text Mining, University of Manchester, Manchester, UK.

${ }^{2}$ Artificial Intelligence Research Center, National Research and Development Agency (AIST), Tokyo, Japan.

\section{References}

1. Wang Y, Ananiadou S, Tsujii J. Improve chinese clinical named entity recognition performance by using the graphical and phonetic feature. In: 2018 IEEE International Conference on Bioinformatics and Biomedicine (BIBM). Piscataway: IEEE; 2018. p. 1582-6.

2. Boltz WG. The Origin and Early Development of the Chinese Writing System, vol. 78. University Park: Eisenbrauns; 1994.

3. Wu Y, Jiang M, Lei J, Xu H. Named entity recognition in chinese clinical text using deep neural network. Stud Health Tech Inf. 2015;216:624.

4. Peng N, Dredze M. Improving named entity recognition for chinese social media with word segmentation representation learning. In: Proceedings of the 54th Annual Meeting of the Association for Computational Linguistics, vol. 2. Stroudsburg, Pennsylvania: ACL; 2016. p. 149-55.

5. Luong T, Socher R, Manning CD. Better word representations with recursive neural networks for morphology. In: CoNLL. Stroudsburg, Pennsylvania: ACL; 2013. p. 104-13.

6. Bojanowski P, Grave E, Joulin A, Mikolov T. Enriching word vectors with subword information. Trans Assoc Comput Linguist. 2017;5:135-46. MIT Press; Cambridge, Massachusetts.

7. Yu J, Jian $X$, Xin $H$, Song Y. Joint embeddings of chinese words, characters, and fine-grained subcharacter components. In: Proceedings of the 2017 Conference on Empirical Methods in Natural Language Processing. Stroudsburg, Pennsylvania: ACL; 2017. p. 286-91.

8. Mikolov T, Chen K, Corrado G, Dean J. Efficient estimation of word representations in vector space. arXiv preprint arXiv:1301.3781. 2013. https://arxiv.org/abs/1301.3781.

9. Cao S, LuW, Zhou J, LiX. cw2vec: Learning Chinese word embeddings with stroke n-gram information. In: Thirty-Second AAAI Conference on Artificial Intelligence. Palo Alto: AAAl; 2018.

10. Dong C, Zhang J, Zong C, Hattori M, Di H. Character-based Istm-crf with radical-level features for chinese named entity recognition. In: International Conference on Computer Processing of Oriental Languages. Berlin: Springer; 2016. p. 239-50.

11. Dai F, Cai Z. Glyph-aware embedding of chinese characters. In: Proceedings of the First Workshop on Subword and Character Level Models in NLP. Stroudsburg: ACL; 2017. p. 64-9.

12. Huang $Z$, Xu W, Yu K. Bidirectional Istm-crf models for sequence tagging arXiv preprint arXiv:1508.01991. 2015. https://arxiv.org/abs/1508.01991.

13. 医学 - 维基百科, 自由的百科全书; . https://zh.wikipedia.org/wiki/ Category:\%E7\%94\%9F\%E7\%89\%A9\%E5\%AD\%A6. Accessed 29 Aug 2018.

14. 生物学 - 维基百科, 自由的百科全书: https://zh.wikipedia.org/wiki/ Category:\%E5\%8C\%BB\%E5\%AD\%A6. Accessed 29 Aug 2018.

15. Google Code Archive - Long-term Storage for Google Code Project Hosting. https://code.google.com/archive/p/word2vec. Accessed 29 Aug 2018.

16. GitHub - HKUST-KnowComp/JWE: Joint Embeddings of Chinese Words, Characters, and Fine-grained Subcharacter Components. https://github. com/HKUST-KnowComp/JWE. Accessed 10 Sept 2018.

17. GitHub - Bamtercelboo/cw2vec: Cw2vec: Learning Chinese Word Embeddings with Stroke N-gram Information. https://github.com/ bamtercelboo/cw2vec. Accessed 10 Sept 2018.

18. Unihan Database Lookup. http://www.unicode.org/charts/unihan.html. Accessed 30 Aug 2018.

19. CHISE Project. http://www.chise.org/index.en.html. Accessed 08 Feb 2019.

\section{Publisher's Note}

Springer Nature remains neutral with regard to jurisdictional claims in published maps and institutional affiliations. 